\title{
Adaptive Reduced-Basis Generation for Reduced-Order Modeling for the Solution of Stochastic Nondestructive Evaluation Problems
}

\author{
Bahram Notghi ${ }^{1}$, Mohammad AhmadPoor $^{2}$, John C. Brigham ${ }^{3}$
}

\begin{abstract}
A novel algorithm for creating a computationally efficient approximation of a system response that is defined by a boundary value problem is presented. More specifically, the approach presented is focused on substantially reducing the computational expense required to approximate the solution of a stochastic partial differential equation, particularly for the purpose of estimating the solution to an associated nondestructive evaluation problem with significant system uncertainty. In order to achieve this computational efficiency, the approach combines reduced-basis reduced-order modeling with a sparse grid collocation surrogate modeling technique to estimate the response of the system of interest with respect to any designated unknown parameters, provided the distributions are known. The reduced-order modeling component includes a novel algorithm for adaptive generation of a data ensemble based on a nested grid technique, to then create the reduced-order basis. The capabilities and potential applicability of the approach presented are displayed through two simulated case studies regarding inverse characterization of material properties for two different physical systems involving some amount of significant uncertainty. The first case study considered characterization of an unknown localized reduction in stiffness of a structure from simulated frequency response function based nondestructive testing. Then, the second case study considered characterization of an unknown temperature-dependent thermal
\end{abstract}

\footnotetext{
${ }^{1}$ Department of Mechanical Engineering, Johns Hopkins University, Baltimore, MD 21218, USA, E-mail: notghi@jhu.edu

${ }^{2}$ Department of Civil and Environmental Engineering, Swanson School of Engineering, University of Pittsburgh, Pittsburgh, PA, USA, E-mail: moa28@pitt.edu

${ }^{3}$ School of Engineering and Computing Sciences, Durham University, UK, E-mail: john.brigham@durham.ac.uk
}

Preprint submitted to Computer Methods in Applied Mechanics and EngineeringJune 19, 2016

C 2016. This manuscript version is made available under the Elsevier user license http://www.elsevier.com/open-access/userlicense/1.0/ 
conductivity of a solid from simulated thermal testing. Overall, the surrogate modeling approach was shown through both simulated examples to provide accurate solution estimates to inverse problems for systems represented by stochastic partial differential equations with a fraction of the typical computational cost.

Keywords:

Material characterization, Nondestructive evaluation, Computational inverse mechanics, Uncertainty quantification, Reduced order model, Sparse grid Collocation

\section{Introduction}

There is a large number of important inverse problems in engineering mechanics, covering applications from material characterization to design of complex physical systems, and a corresponding large amount of work involving the solution of these problems. Of particular interest to the present work are inverse problems relating to the use of nondestructive evaluation (NDE) to evaluate various properties of different in-service structures/systems $[4,8$, $40,50]$. One effective approach for solving such inverse problems in mechanics has been to use a numerical analysis tool, such as finite element analysis, to predict the forward response of the system under consideration and then combine nonlinear optimization to find the unknown properties to best match the response of the numerical model with the desired or measured response of the system $[2,51,17,38,39,25]$. Although various research efforts have been directed towards such computational methods for the solution of inverse problems and have made significant strides, there are still several common challenges, most often relating to the ill-posedness of the inverse problems in the form of nonexistent or non-unique solutions along with the excessive computational expense associated with many solution algorithms. In particular, regardless of the solution approach (e.g., gradient based [37, 31] or nongradient-based optimization [14, 23, 45], etc.), solving a NDE problem using a computational solution procedure commonly requires a relatively large number of evaluations of the forward response of the system. Moreover, the computational expense drastically increases if considering uncertainty within the system, since the forward problem then involves a stochastic partial differential equation (SPDE) (which is considerably expensive on its own).

There are several different approaches that have been developed for the 
solution of SPDEs $[22,9,54]$. Whether the approach to address the uncertainty is intrusive (i.e., modifies the deterministic boundary value problem) or non-intrusive (i.e., only uses results from the deterministic boundary value problem), these solution approaches typically require a substantial amount of computational expense. As such, there has been considerable effort to attempt to reduce the computational expense of SPDE solutions, both for intrusive $[12,47,6,44]$ and non-intrusive approaches [26, 27]. Sparse grid approximation approaches are one particular computationally efficient solution technique that builds a low-cost approximation (i.e., surrogate model) of the SPDE and has shown considerable promise for being used in approximating SPDE solutions $[48,54]$. The sparse grid methods are non-intrusive, and therefore, easy to implement, requiring only the solution of uncoupled deterministic problems, and use substantially fewer evaluations of the boundary value problem in comparison to the traditional Monte Carlo non-intrusive methods, without sacrificing accuracy [9].

There have also been a variety of approaches developed in recent years to solve inverse problem involving SPDEs (i.e., stochastic inverse problems). For example, [35] presented an approach to solve a stochastic inverse heat conduction problem using the spectral stochastic finite element method as the forward solver (i.e., to solve the SPDEs) within an optimization routine. The work in [21] used the sparse grid collocation method based on the Smolyak algorithm with adaptive refinement based on the importantance of the stochastic dimensions to solve stochastic natural convection problems. This work was extended for solving a design problem by using a sparse grid representation of the design variables to convert the stochastic optimization problem into a deterministic optimization problem, and gradient-based optimization was used to solve the design problem with stochastic sensitivity computation [55]. In order to reduce the computational cost for Bayesian solutions of inverse problems, Marzouk et al. [34] introduced a method that combines Karhunen-Love (K-L) representation of the unknown field with spectral methods, in which the K-L representation of a scaled Gaussian process prior defines the uncertainty that is propagated through the forward model with a stochastic Galerkin scheme. More recently, Marzouk et al. [33] presented an efficient numerical method, which used generalized polynomial chaos (gPC) to construct a polynomial approximation of a forward solution and the support of the prior distribution to define a surrogate posterior probability density that can be evaluated at low computational cost.

Reduced-basis-type model reduction approaches that identify the rela- 
tively low-dimensional basis that is optimal in some sense for representing the physics of the system of interest have been used to produce efficient and accurate numerical representations for several different applications in mechanics $[24,18,41,13,3]$. By not replacing the boundary value problem governing the mechanics of interest as would be done with surrogate modeling approaches, reduced-basis ROM techniques are more computationally expensive than surrogate modeling approaches, but are typically capable of more accurate approximations, particularly for extrapolating throughout the space of potential system inputs. This ROM approach has also been recently extended to stochastic problems with the work by Boyaval et al. [12] that created reduced-basis ROMs to estimate the solution of an SPDE. There are different strategies to determine the low-dimensional basis, but the focus of the work herein is on methods that derive the "optimal" basis from a given set of potential fields for the system of interest. These given fields can be either experimentally measured or numerically simulated with different values of the system input parameters, depending on capabilities. There are also different approaches to process these given fields to produce a basis. For example, proper orthogonal decomposition (POD) has been used in several studies $[7,29,1]$ to extract the basis from a given dataset that is optimal in the average $L_{2}$-error sense for representing the given fields. Alternatively, other works, including the work by Boyaval et al. relating to SPDEs, have simply used Gram Schmidt orthogonalization to directly convert the given fields into an orthogonal basis [12]. Yet, a more important question that is not often addressed in ROM studies is how to generate (e.g., select the system inputs to numerically simulate with full-order analysis) the initial set of potential fields used to create the basis. The majority of the previous work has used some form of fixed sampling, often simply uniformly sampling the input parameter space [30]. Alternatively, one approach that has been developed and referred to as "certified reduced basis methods" uses a posteriori error estimation to iteratively add to a set of potential fields to minimize the error of the resulting ROM with respect to the estimated error bound [43]. However, the ability to generate and ensure accuracy of the a posteriori error estimate for the ROM with a given PDE can be nontrivial and a potentially costly task. A different approach was presented by Brigham and Aquino [13] to generate the dataset for ROM creation that was based on creating the set of potential fields that were maximally diverse in a sense within the solution space. However, this maximum diversity approach was only applicable as presented for problems relating to solid mechanics of rate-dependent mate- 
rials. Overall, no clear approach exists as of yet that is generally applicable, easy to implement, and computationally inexpensive to generate a suitable dataset and ultimately an accurate ROM.

The current work presents an approach to substantially reduce the computational expense required to approximate the solution of a stochastic PDE, particularly for the purpose of estimating the solution to an associated NDE problem. In order to achieve this computational efficiency, the approach presented combines reduced-basis reduced-order modeling with a sparse grid collocation surrogate modeling technique to estimate the response of the system of interest with respect to both the inverse problem unknowns and the uncertain system parameters. One of the main features of the proposed method is the use of the nested properties of the grid for collocation to avoid the computational expense when expanding the number of samples and regenerating the reduced-order model to obtain higher accuracy. In addition, the same full-order models used for generating the reduced-order model are used to construct the Sparse grid approximation. In the following section a general inverse problem solution framework is outlined. Then, the approach for using the sparse grid method as a system response surrogate model is presented, which is followed by an adaptive method for generating efficient and accurate reduced-order models for the solution of SPDEs. Lastly, the capability of this computationally efficient approach to provide accurate solution estimates to material characterization problems of systems represented by SPDEs with a fraction of the typical computational cost is shown through simulated examples involving both solid mechanics and heat transfer.

\section{Methods}

For context, the methods are presented with respect to nondestructive evaluation problems based upon some type of physical system measurements (e.g., displacements or temperatures) given the associated boundary conditions and some knowledge of significant epistemic uncertainty in certain system parameters. Furthermore, the present work utilizes a standard generallyapplicable optimization-based computational approach for inverse problem solution approximation to evaluate the ROM strategy. As is typical, the computational inverse mechanics approach consists of first constructing a numerical representation of the behavior of the target system that is parameterized with respect to the unknown system properties. Then, an objective functional is constructed that quantifies the difference between the measured 
response and the response predicted by the numerical representation for any given admissible set of system properties. Lastly, all that is necessary is to minimize the objective functional with respect to the unknown system properties to produce an estimate to the inverse problem solution. As an example, a common objective functional for the inverse solution approach could have the following form:

$$
J(\vec{h})=\frac{\left\|R^{m e s}-R(\vec{c}, \vec{h})\right\|}{\left\|R^{m e s}\right\|},
$$

where $\vec{h}$ is the vector of unknown parameters (e.g. parameters defining a material property distribution) to be determined through the inverse solution process, $R^{m e s}$ is the measured response of the system, $R$ is the simulated estimate to the system's response for a given parameter set estimate, $\vec{c}$ is the vector of uncertain system parameters, and $\|$.$\| is some suitable metric norm$ that combines the contributions of all measurement information to produce the total scalar error functional. The components of $\vec{c}$ are assumed to belong to an event space of a standard complete probability space $[32,54]$.

A critical point is that the calculation of the simulated response of the structure with respect to different parameter estimates using traditional analysis methods (e.g., finite element analysis) can be computationally expensive for many realistic applications, which can lead to the inverse solution process becoming computationally infeasible (especially when addressing uncertainty). Therefore, rather than using computationally expensive methods to numerically simulate the system response estimate, the present work instead utilizes a substantially more computationally inexpensive surrogate modeling strategy to produce a tool to simulate the system response $(R)$ with negligible computational expense. In particular, this work presents a combined ROM-collocation strategy to effectively and efficiently generate a surrogate model of the system response with respect to both the inverse problem unknowns and the uncertain system parameters with the assumption that the distributions of the unknown parameters is known. As outlined in the following, this approach not only uses a collocation method to obtain the surrogate model for the system response, but generates and uses a ROM to create this surrogate model. This combination provides a substantial savings in computational expense in comparison to traditional techniques at each step in the process and for the overall solution procedure. 


\subsection{Sparse Grid Collocation Method for Forward Model Approximation}

A sparse grid collocation method was selected to create the system response surrogate model for the present work due to its capabilities to provide accurate approximations of smooth functions in high dimensions based on a relatively small number of function evaluations, as has been shown in several works relating to global optimization [20]. For the purposes of the sparse grid collocation method, the vector of unknown/inverse problem parameters $(\vec{h})$ and the vector of uncertain system parameters $(\vec{c})$ are treated equivalently, and therefore, are combined into a single parameter vector, $\vec{\varphi}=[\vec{h}, \vec{c}]^{T}$, for this presentation.

The basic collocation approach uses Lagrange interpolating polynomials and the tensor product technique to incorporate all dimensions of the parameter space to define the surrogate model approximation of the system response in terms of a higher-order (e.g., finite element analysis) model of the system response evaluated at each collocation point in the parameter space as:

$$
\left(U^{i_{1}} \otimes \cdots \otimes U^{i_{n}}\right)\left(R^{H O}\right)=\sum_{j_{1}=1}^{m_{1}} \cdots \sum_{j_{n}=1}^{m_{n}} R^{H O}\left(\varphi_{j_{1}}^{i_{1}}, \ldots, \varphi_{j_{n}}^{i_{n}}\right) \cdot\left(L_{j_{1}}^{i_{1}} \otimes \cdots \otimes L_{j_{n}}^{i_{n}}\right)
$$

where $R^{H O}$ is the higher-order model response, $L_{j}^{i}$ is the $j^{\text {th }}$ standard Lagrange interpolating polynomial corresponding to the $i^{\text {th }}$ parameter and $m_{i}$ is the number of collocation points for the $i^{\text {th }}$ parameter. Note that each parameter is typically mapped to a domain of $[-1,1]$ for this implementation. While this collocation approach is a relatively straightforward technique overall, the selection of the collocation points is a nontrivial task. With the total number of evaluations of the higher-order model of the system response being equivalent to $m_{1} \times \cdots \times m_{n}$, the creation of the surrogate model by simply using uniformly spaced collocation points in the parameter space can be excessively computationally expensive, even for a relatively inexpensive higher-order model $\left(R^{H O}\right)$. Thus, the sparse grid approach is used here to reduce the computational cost by significantly reducing the total number of required collocation points without significantly sacrificing accuracy of the surrogate model.

For the present study, the Smolyak algorithm [52, 10, 46] was used for the sparse grid construction. At its core, the Smolyak algorithm creates a 
sparse grid interpolant to be utilized in place of the tensor product above as:

$$
A_{q, n}=\sum_{q-n+1 \leq|\vec{i}| \leq q}(-1)^{q-|\vec{i}|} \cdot\left(\begin{array}{c}
n-1 \\
q-|\vec{i}|
\end{array}\right) \cdot\left(U^{i_{1}} \otimes \cdots \otimes U^{i_{d}}\right),
$$

where $n$ is the total number of system parameters (i.e., dimension of $\vec{\varphi}$ ), $q-n$ is the order of interpolation, $\vec{i}=\left(i_{1}, \ldots, i_{n}\right)$, and $|\vec{i}|=i_{1}+\cdots+i_{n}$ (note that $i_{k}$ conceptually represents the level of interpolation along the the $k^{\text {th }}$ direction). To compute the surrogate model response approximation, $R=A_{q, n}\left(R^{H O}\right)$, the response function should be evaluated at the sparse grid points given by

$$
\mathscr{H}_{q, n}=\bigcup_{q-n+1 \leq|i| \leq q}\left(\Phi^{i_{1}} \otimes \cdots \otimes \Phi^{i_{n}}\right)
$$

where $\Phi^{i_{k}}=\left(\varphi_{1}^{i_{k}}, \ldots, \varphi_{m_{k}}^{i_{k}}\right)$. Lastly, there are different potential choices for the nested grid to define the collocation points in the parameter space, such as the Gauss Patterson approach [15] or the Clenshaw-Curtis approach [49].

Utilizing a sparse grid approximation allows for construction of the surrogate model with orders of magnitude reduction in the number of higher-order analyses required compared to a standard tensor product implementation with approximately the same level of accuracy. However, sparse grids are still affected by the "curse of dimensionality," and the number of analyses required for relatively high-dimensional parameter spaces can become excessive if the higher-order model being approximated requires a substantial computational expense (as would often be the case if using traditional finite element analysis to produce the system response here). Thus, the present works adds one more layer of computational savings by building and using a ROM (instead of a commonly used full-order finite element analysis) for the higher-order analysis in the surrogate model creation, as detailed in the following.

\subsection{Adaptive Nested Sampling for Reduced-Order Model Generation}

The reduced-order modeling strategy utilized herein is the reduced-basis approach, which in essence, identifies the low-dimensional basis that is optimal in some sense to replace the standard higher-order generalized (e.g., polynomial) bases typically used within a numerical PDE solution strategy (e.g., weak form Galerkin finite element method)[42]. This approach was chosen since it balances the improvement in computational expense with the 
capability to maintain accurate generalization over the parameter space that is afforded by maintaining the physics of the problem through the PDE, which is still included in the solution procedure, in contrast to alternate ROM techniques. The following discussion of an approach to create such an accurate physics-based ROM is presented in a general format, which can be used for a variety of mechanics, including both steady-state and transient processes, etc., and could even be applicable as a direct replacement in a computational inverse problem solution procedure (although, that was not the focus of the work herein).

To understand the ROM approach, first consider the objective being to find a stochastic function (the primary system response field), $\vec{u} \equiv \vec{u}(\vec{x}, t, \vec{\varphi}) \in$ $\Omega \times D$, with $\vec{\varphi} \in D$, with the assumption that $\vec{u}$ is a sufficiently smooth function, such that a sparse grid approximation is applicable (a detailed discussion regarding the smoothness requirements of the solution can be found in [15]). Then, the following general form of a SPDE representing the behavior of a system of interest (arbitrarily shown as transient and with only essential boundary conditions for clarity, but could equivalently be utilized for a static problem, a problem in the frequency domain, and/or a problem with other boundary conditions as well) holds:

$$
\begin{array}{r}
\frac{\partial^{n} \vec{u}(\vec{x}, t ; \vec{\varphi})}{\partial t^{n}}=F(\vec{\varphi} ; \vec{u}(\vec{x}, t ; \vec{\varphi})), \quad \forall \vec{x} \in \Omega \\
\frac{\partial^{r} \vec{u}(\vec{x}, t=0 ; \vec{\varphi})}{\partial t^{r}}=g_{r}(\vec{x} ; \vec{\varphi}), \quad \forall \vec{x} \in \Omega, \text { for } r=0, \ldots, n-1 \\
\vec{u}(\vec{x}, t ; \vec{\varphi})=\vec{u}^{0}(\vec{x}, t ; \vec{\varphi}) \quad \forall \vec{x} \in \Gamma,
\end{array}
$$

where $\vec{x}$ is the spatial position, $t$ is time, $F$ is a spatial differential operator, $n$ is the number of temporal derivatives, $g_{r}$ are the known initial conditions, $\vec{u}^{0}$ is the known boundary condition, $\Omega$ is the spatial domain, and $\Gamma$ is the domain boundary. The core hypothesis of the reduced-basis reduced-order modeling approach considered in the present work is that a relatively small number of full-order (i.e., traditional finite element) analyses based upon different values of the input parameters of interest $(\vec{\varphi})$ contain fundamental information about the spatial distribution of potential solution fields of the boundary value problem (BVP) and can be used to derive a low-dimensional basis that can predict the solution fields for a range of input parameters (not just the specific parameter values used to generate the set of full-order analyses) with reasonable accuracy. Recall that $\vec{\varphi}$ contains both the vector of unknown/inverse problem parameters and the vector of uncertain system 
parameters. Also, note that the nature of the distribution of each uncertain system parameters (e.g., uniform, normal, etc.) is assumed to be known $a$ priori.

The proper orthogonal decomposition (POD) method was used herein to derive the basis to be used from a set of previously generated full-order analysis solution fields [11]. As detailed in [13, 28, 11], POD creates the basis that is optimal in an $L_{2}$ average sense for approximating the given solution fields. Assuming such an $m$-dimensional basis has been created, the solution of the SPDE (Equation 5) can be approximated by a linear combination of the basis functions (i.e., modes) as:

$$
\vec{u}(\vec{x}, t ; \vec{\varphi})=\sum_{i=1}^{m} a_{i}(t ; \vec{\varphi}) \vec{\phi}_{i}(\vec{x})
$$

where $a_{i}(t ; \vec{\varphi})$ is the $i^{t h}$ modal coefficient to be determined by the numerical analysis to approximate the solution of the system given a new set of system input parameters, whether those parameters are unknowns to be determined through an inverse solution process or other uncertain parameters of the system. Applying the Galerkin projection procedure, the solution approximation (Equation 6) can be substituted into the governing SPDE (Equation 5) to obtain the weak form of the SPDE that can be used to approximate the modal coefficients as:

$$
\frac{\partial^{n} \alpha_{c}(t ; \vec{\varphi})}{\partial t^{n}}=\left(F\left(\vec{\varphi} ; \sum_{i=1}^{N} a_{i}(t ; \vec{\varphi}) \vec{\phi}_{i}(\vec{x})\right), \vec{\phi}_{c}(\vec{x})\right)_{L_{2}}, \quad \text { for } c=1, \ldots, m
$$

with the initial conditions given as:

$$
\alpha_{c}(t=0 ; \vec{\varphi})=\left(g_{r}(\vec{x} ; \vec{\varphi}), \phi_{c}\right)_{L_{2}}, \quad \text { for } r=0, \ldots, n-1, \quad c=1, . ., m,
$$

where $(\cdot, \cdot)_{L_{2}}$ is the standard $L_{2}$ inner product. Note that for the case of fixed essential boundary conditions, the modes will automatically satisfy the essential boundary conditions for the system of interest.

Of paramount importance is that a critical question still remains unanswered from the above formulation, which is how to select the set of input parameters used to create the set of full-order analyses that will be utilized to then create the POD basis and ultimately the ROM. In particular, to see a true benefit from this ROM strategy, this dataset must be generated in 
such a way to limit the number of full-order simulations necessary to ensure sufficiently accurate generalization of the ROM over the admissible range of the input parameters of interest. As such, the following presents an adaptive strategy to incrementally select the parameter set values to be used to create full-order analysis fields (referred to as "snapshots" from here on) and build the ROM to minimize a measure of the potential ROM solution error.

\subsubsection{Adaptive Nested Grid Snapshot Generation:}

The core of this incremental snapshot generation approach is the use of a nested grid strategy to select the associated parameter set values along with a local refinement procedure to select additional parameter set values adaptively in the region of the parameter space with the highest approximation error. The assumption is that this point of highest error indicates a region of inaccuracy within the sparsely sampled space, that should therefore be adaptively resampled (including the point with highest error itself) to best improve the approximation capability. In addition, the parameter space sampling that is used to create the ROM can be done in such a way (as was done herein) that a portion of the parameter space grid points corresponding to the snapshots are the exact same points as a portion of the collocation points that will then be used to create the surrogate model (as described in Section 2.1). Since, the points in the parameter space corresponding to the full-order analyses are naturally the most accurate (in the ROM context), having the grids overlap in this two stage (i.e., ROM to surrogate model) process will ensure that the information with the highest possible accuracy is used to create the numerical representation of the system response that will ultimately be used in the inverse problem solution procedure.

The algorithm developed for adaptive nested grid snapshot generation is outlined in Algorithm 1. For the present work, an ad hoc approach was used to estimate the approximation error of the ROM based on a randomly generated set of full-order analysis response fields (i.e., "ROM test" set) to drive the adaptive snapshot generation procedure. Two different measures of this approximation error are utilized at two different stages in the snapshot generation algorithm, the average relative $L_{2}$-error, which is defined as:

$$
E_{L_{2}}^{a v e}(R O M)=\frac{1}{a} \sum_{k=1}^{a} \frac{\left\|R^{F O M}\left(\vec{x}, \vec{\gamma}_{k}\right)-R^{R O M}\left(\vec{x}, \vec{\gamma}_{k}\right)\right\|_{L_{2}(\Omega)}}{\left\|R^{F O M}\left(\vec{x}, \vec{\gamma}_{k}\right)\right\|_{L_{2}(\Omega)}}
$$




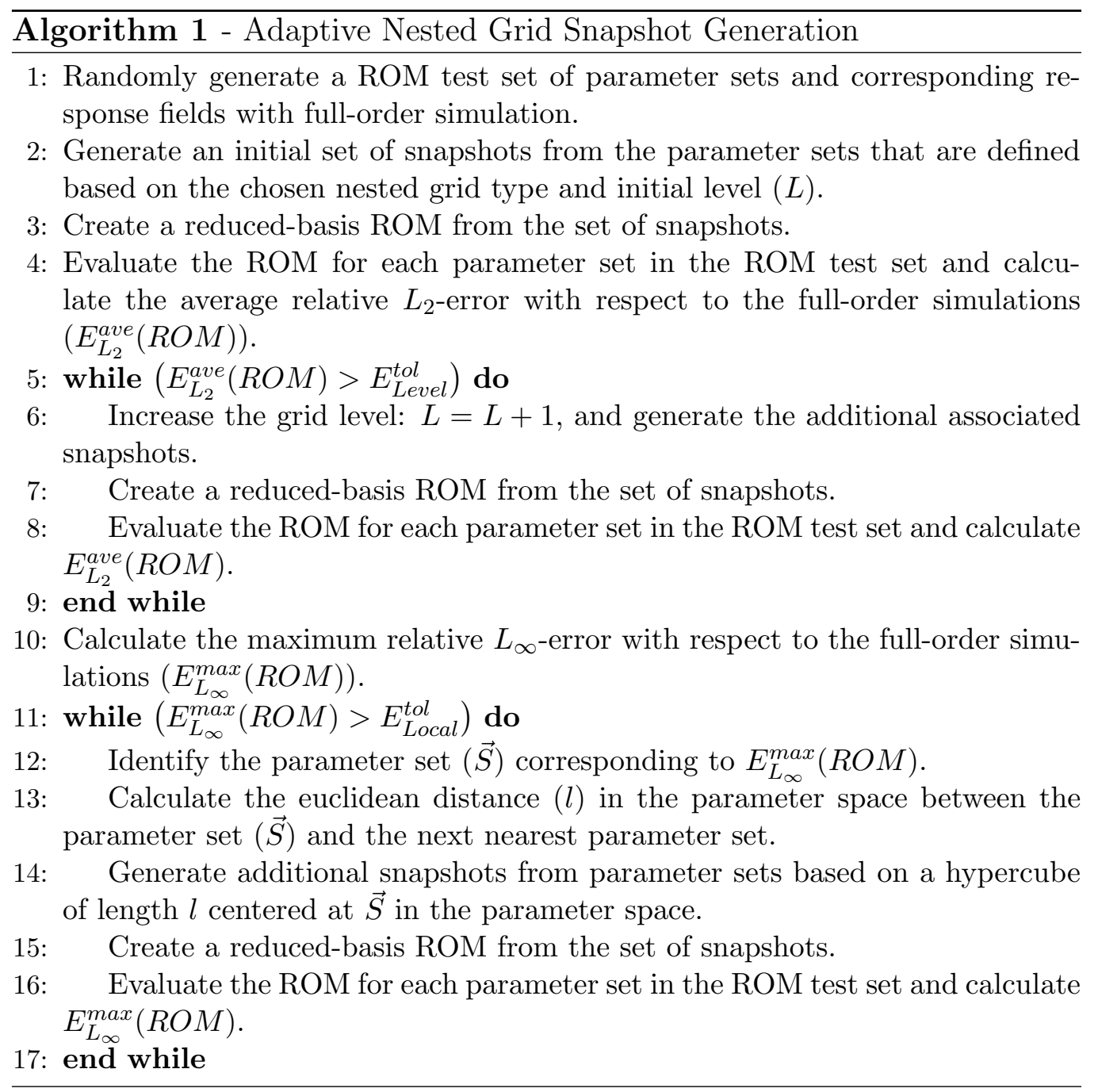


and the maximum relative $L_{\infty}$-error, which is defined as:

$$
E_{L_{\infty}}^{\max }(R O M)=\operatorname{Max}_{k \in[1, a]} \frac{\left\|R^{F O M}\left(\vec{x}, \vec{\gamma}_{k}\right)-R^{R O M}\left(\vec{x}, \vec{\gamma}_{k}\right)\right\|_{L_{\infty}(\Omega)}}{\left\|R^{F O M}\left(\vec{x}, \vec{\gamma}_{k}\right)\right\|_{L_{\infty}(\Omega)}},
$$

where $a$ is the number of response fields in the ROM test set, $\vec{\gamma}_{k}$ is the set of all nondestructive test parameters that the measurements are obtained from (e.g., excitation frequency, sensor location, time, etc.), $R^{F O M}$ is the response field simulated with the full-order model, $R^{R O M}$ is the response field simulated with the ROM, and $\|\cdot\|_{L_{2}(\Omega)}$ and $\|\cdot\|_{L_{\infty}(\Omega)}$ are the standard $L_{2}$ and $L_{\infty}$ norms, respectively. In addition, two error tolerance values are set by the user, $E_{\text {Level }}^{\text {tol }}$ (i.e., the level error tolerance), which corresponds to the average relative $L_{2}$-error and is used to determine the final level of the nested grid to generate the snapshots, and $E_{\text {Local }}^{\text {tol }}$ (i.e., the local error tolerance), which corresponds to the maximum relative $L_{\infty}$-error and is used during the local refinement process. Therefore, the first step in the snapshot generation procedure is to create the ROM test set by randomly generating a set of parameter sets and calculating the associated response fields with the full-order simulation. Then, an initial set of snapshots is created based on a nested grid with a selected level (this level could typically be chosen as an arbitrarily small number, such as 1) and an ROM is created from this set of snapshots. The level of the nested grid is iteratively increased, with the corresponding new parameter sets evaluated with the full-order model and the ROM updated after each iteration until the average relative $L_{2^{-}}$ error of the ROM computed with the ROM test set is below the level error tolerance. In order to improve the accuracy of the ROM in the regions of the parameters space that highest error metric, the local refinement procedure iteratively generates additional snapshots by sampling a hypercube with a user-defined sampling procedure (e.g., a uniform grid) in the parameter space of user-defined relative length $(l)$ centered at the parameter set values from the ROM test set corresponding to the maximum relative $L_{\infty}$-error $(\vec{S})$, and the ROM is again updated after each iteration until the maximum relative $L_{\infty}$-error is below the local error tolerance. One of the choices for userdefined relative length $(l)$ in the local refinement process can be defined as the euclidean distance in the parameter space between the parameter set corresponding to $E_{L_{\infty}}^{\max }(R O M)(\vec{S})$ and the next nearest parameter set from the current set of snapshots used to create the ROM. 


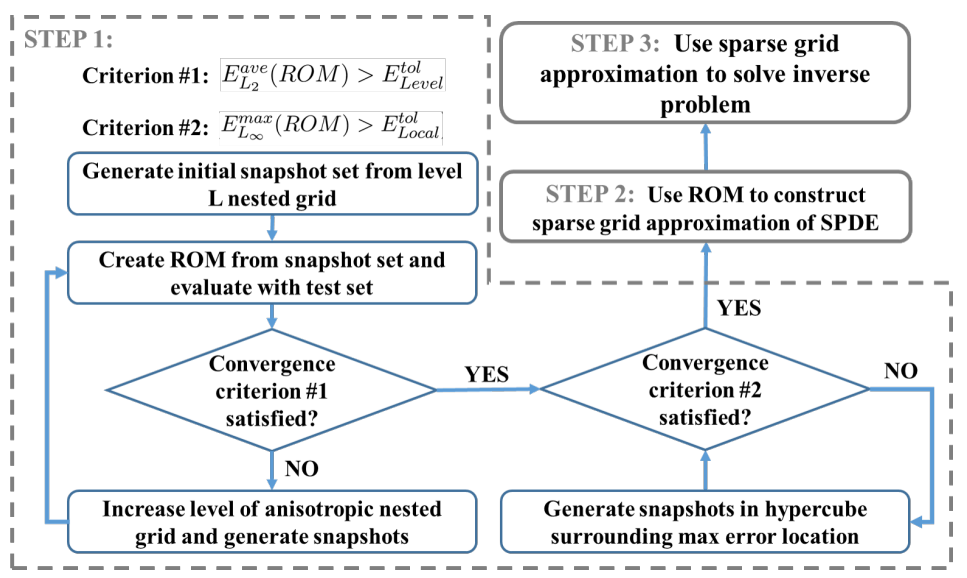

Figure 1: Flow chart for computationally efficient approximation of stochastic NDE problems.

\subsection{Computationally Efficient Stochastic Nondestructive Evaluation Proce- dure}

Putting all of the components together, the complete process for computationally efficient approximation of NDE problems involving SPDEs is as following (shown schematically in Figure 1):

Step 1: Create a reduced-order model for the SPDE of interest using a traditional (full-order) numerical solution technique (e.g., finite element anlaysis) to adaptively generate an optimal set of snapshots (Section 2.2).

Step 2: Create a surrogate model for the SPDE of interest that will estimate the system response given inverse problem and uncertain system parameters using the reduced-order model with the sparse grid collocation method (Section 2.1).

Step 3: Apply the computational inverse problem solution procedure to estimate the solution to the inverse problem by minimizing the difference between the measured/target system response and the response estimated by the surrogate model (Equation (1)).

\section{Example and Discussion}

Two numerical case studies were considered to investigate the potential capabilities of the presented approach for creating a ROM using an adaptive 
nested grid sampling strategy and utilizing this ROM to construct a surrogate model to be used in a NDE problem with significant system uncertainty. The objective of the first case study was to inversely calculate the unknown localized reduction in stiffness of a structure from simulated frequency response function based nondestructive testing. Alternatively, the objective of the second case study was to inversely calculate the temperature-dependent thermal conductivity of a solid from simulated thermal testing.

For both case studies standard Galerkin finite element analysis [42] was used to simulate the "experimental" measurements and to generate the snapshots for the ROM construction. For all examples, the Gauss Patterson approach $[49,16]$ was chosen to define the grid points for both the snapshot selection process to create ROMs and the sparse grid surrogate model method. The ROM test sets for calculating the error norms of the ROM were comprised of 50 generated response field snapshots based on low-discrepancy quasi-random Hammersley sampling methods [36, 53], the level error tolerance was defined as $E_{\text {Level }}^{\text {tol }}=5 \%$, and the local error tolerance was defined as $E_{\text {Local }}^{\text {tol }}=1 \%$ for both example. Gauss Patterson grids were used for the sparse grids with an initial level of one, and the local refinement process for the ROM generation added 5 sample points inside an adaptively defined hypercube with length $(l)$, in which $l$ was chosen as the euclidean distance in the parameter space between the parameter set corresponding to $E_{L_{\infty}}^{\max }(R O M)$ $(\vec{S})$ and the next nearest parameter set from the current set of snapshots used to create the ROM. A genetic algorithm, specifically the NSGA-II by Deb. [19] (a stochastic optimization algorithm) was applied to approximate the inverse problem solutions for all examples (as described in Section 2). For simplicity, standard GA parameters were utilized and the stopping criteria was set to be well in excess of the actual convergence of the GA population to ensure that the optimization process was as successful as possible for each trial, and therefore, did not bias the results. An important point is that although a GA provides significant global search capabilities, a GA would typically be computationally excessive for problems such as this, since GAs usually require a relatively large number of function evaluations to converge to a solution. However, the use of surrogate modeling herein allows even a GA to be applied with relatively low overall computational expense for the inverse solution process. An addition note is that to add some amount of realism to the examples and to partially relieve the inverse crime inherent in simulated experiments, $1 \%$ Gaussian white noise was added to all simu- 


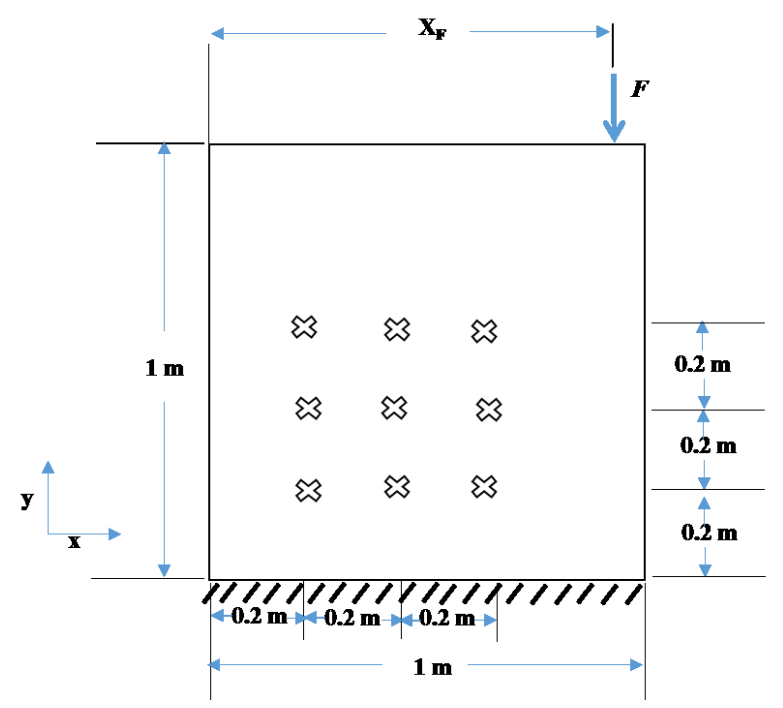

Figure 2: Schematic for the first example of stiffness characterization of a plate from frequency response testing, displaying the sensor locations (x-marks) and the actuator location $\left(X_{F}\right)$.

lated experimental responses prior to applying the inverse characterization procedure as:

$$
R^{m e s}=R_{0}^{m e s}(1+0.01 \aleph),
$$

where $R_{0}^{\text {mes }}$ is the original simulated test response without noise and $\aleph$ is a normally distributed random variable with zero mean and unit variance.

\subsection{Stiffness Characterization from Frequency Response Testing}

The first numerical example was based upon characterization of the stiffness of a solid from frequency response testing and consisted of a $1 \mathrm{~m} \times 1 \mathrm{~m} \times$ $0.01 \mathrm{~m}$ aluminum plate, that was fixed along the bottom and free to displace on the remaining three sides. Figure 2 shows a schematic of the plate and the simulated nondestructive test used to perform the material characterization. For the simulated test, an actuator was assumed to be represented by a harmonic $2 \mathrm{D}$ pressure force $\mathrm{F}=(50 \mathrm{~N} / \mathrm{m}) \times \sin (\omega t)$ applied to a $0.05 \mathrm{~m}$ region normal to the top surface that excited the structure to steady state at 10 equally spaced excitation frequencies between 100 to $1000 \mathrm{~Hz}$, while the resulting harmonic displacement amplitudes were measured at nine "sensor" locations for each excitation frequency. The horizontal position of the actuator (defined as the location of the resultant of the distributed load) was 
assumed to be the main source of uncertainty for this inverse problem example, and was assumed to be described by a uniformly distributed random variable $X_{F} \sim U(0.625,0.825) m$.

\subsubsection{Forward Problem and Reduced-Order Modeling:}

The structure was assumed to behave linearly elastically with respect to the nondestructive testing (NDT) and represented by steady-state dynamic solid mechanics. Therefore, the SPDE and boundary conditions governing the behavior of the structure can be written as:

$$
\begin{array}{r}
\nabla \cdot \sigma(\vec{x}, \omega, \vec{\varphi})+\omega^{2} \rho \vec{u}(\vec{x}, \omega, \vec{\varphi})=\overrightarrow{0}, \quad \forall \vec{x} \in \Omega, \\
\sigma(\vec{x}, \omega, \vec{\varphi}) \cdot \vec{n}(\vec{x})=\vec{G}(\vec{x}, \omega, \vec{\varphi}), \quad \forall \vec{x} \in \Gamma_{G}, \\
\vec{u}(\vec{x}, \omega)=\vec{u}^{0}(\vec{x}, \omega), \quad \forall \vec{x} \in \Gamma_{u},
\end{array}
$$

where $\sigma$ is the stress tensor, $\omega$ is the excitation frequency, $\vec{\varphi}$ is again the vector of both the unknown/inverse problem parameters (stiffness distribution parameters for this example) and the uncertain system parameters (actuation location for this example), $\rho$ is the density, $\vec{u}$ is the displacement vector, $\vec{n}$ is the unit normal to the surface, $\vec{G}$ is the applied traction vector, $\vec{u}^{0}$ is the vector of applied displacement boundary conditions, $\Omega$ is the domain of the structure, $\Gamma_{G}$ is the portion of the domain boundary with applied traction boundary conditions (which is uncertain for this example), and $\Gamma_{u}$ is the portion of the domain boundary with applied displacement boundary conditions. In addition, the response of the thin plate was assumed to obey the plane stress condition. The material parameters of the plate were based upon standard values for aluminum, with a Poisson's ratio of $\nu=0.3$ and density of $\rho=2700 \mathrm{~kg} / \mathrm{m}^{3}$. The elastic modulus distribution was assumed to be the primary unknown of the inverse problem, but for simplicity, the distribution was assumed to be known to be localized (as could be potentially applicable for damage characterization problems) with a base value of $69 \mathrm{GPa}$, and defined by a radial basis function $(\mathrm{RBF})$ representation as:

$$
E(\vec{x})=\left[1-D \cdot \exp \left(-\frac{\|\vec{x}-\vec{\epsilon}\|^{2}}{c}\right)\right] \times 69 G P a,
$$

where $\|$.$\| represents the standard l_{2}$-norm, $D$ is the Young's modulus percent reduction, $\vec{\epsilon}$, is the center of the RBF (i.e., localized stiffness change), and $c$ is the breadth of the RBF. For the ROM and surrogate model generation and 
the inverse characterization process, the material parameters were assumed to be bounded as follows: $D \in[0,1], \vec{\epsilon} \in[0,1] m \times[0,1] m$, and $c \in[0,0.1] m^{2}$.

Applying the Galerkin projection described in Section 2.2 to the governing equations (Equation 7) produces the ROM for the steady-state dynamic solid mechanics problem as:

$$
\begin{array}{r}
\int_{\Omega} \nabla \overrightarrow{\phi_{c}}(\vec{x}):\left(\mu\left[\sum_{i=1}^{m} \alpha_{i}(\omega, \vec{\varphi}) \nabla \vec{\phi}_{i}(\vec{x})+\left(\sum_{i=1}^{m} \alpha_{i}(\omega, \vec{\varphi}) \nabla \vec{\phi}_{i}(\vec{x})\right)^{T}\right)\right) d \vec{x} \\
+\int_{\Omega} \nabla \overrightarrow{\phi_{c}}(\vec{x}):\left(\lambda\left(\nabla \cdot \sum_{i=1}^{m} \alpha_{i}(\omega, \vec{\varphi}) \vec{\phi}_{i}(\vec{x})\right) I\right) d \vec{x} \\
-\int_{\Omega} \omega^{2} \rho \overrightarrow{\phi_{c}}(\vec{x}) \cdot\left(\sum_{i=1}^{m} \alpha_{i}(\omega, \vec{\varphi}) \vec{\phi}_{i}(\vec{x})\right) d \vec{x} \\
-\int_{\Gamma_{G}} \vec{\phi}_{c}(\vec{x}) \cdot \vec{G}(\vec{x}, \omega, \vec{\varphi}) d \vec{x}=0, \text { for } c=1 \ldots m
\end{array}
$$

where I is the identity tensor, and the Lamé constants can be expressed in terms of the elastic modulus and Poisson's ratio as:

$$
\begin{gathered}
\lambda=\frac{E(\vec{x}) \nu}{(1-2 \nu)(1+\nu)} \\
\mu=\frac{E(\vec{x})}{2(1+\nu)} .
\end{gathered}
$$

The POD procedure with adaptive snapshot generation described in Section 2.2 was applied to determine the basis for the above ROM. As such, snapshot displacement fields were generated with full-order analyses based upon variations in the input parameters of the $\operatorname{RBF}(D, c$ and $\vec{\epsilon})$, the actuation location $\left(X_{F}\right)$, and excitation frequency $(\omega)$. It should be noted that even though the excitation frequency $(\omega)$ was discretely sampled in the simulated test procedure it was treated as a continuous variable identically to the other input parameters with a range of $[100-1000] H z$ for generality in testing the ROM algorithm presented. The adaptive nested grid snapshot generation algorithm converged at a total of 127 snapshots, which were used to create the ROM that would be applied for the surrogate model generation. The final value of $\mathrm{L}$ at convergence was 2 , which produced 97 6-dimensional snapshot parameter sets, and an additional 30 snapshot parameter sets were 
added in the refinement cycle (6 iterations with 5 parameter sets generated each iteration).

An intermediate test was performed first, in which the response approximation capabilities of an ROM created with the adaptive strategy presented was compared to the approximation capabilities of a "baseline" ROM created with an equivalent number (i.e., 127) of snapshots that were entirely randomly generated from the space of input parameters (as has often been the default snapshot creation strategy in the literature). The average relative $L_{2}$-error and the maximum relative $L_{\infty}$-error (as defined previously in 9 and 10, respectively) between both ROMs and a set of 50 test response fields that were generated based on quasi-random low-discrepancy Hammersley sampling methods (and different than the snapshot sets used to generate the ROMs) were calculated. The two error values $\left(L_{2}\right.$ and $\left.L_{\infty}\right)$ for the adaptively generated ROM were $1.09 \%$ and $1.64 \%$, respectively, while the error values for the baseline ROM were $1.97 \%$ and $2.08 \%$, respectively. Thus, the use of the adaptive nested grid snapshot generation strategy improved the accuracy of the resulting $\mathrm{ROM}$ by $46 \%$ in terms of the average relative $L_{2}$-error and $21 \%$ in terms of the $L_{\infty}$-error in comparison to a common random generation approach. In other words, the use of the adaptive snapshot generation procedure to more "intelligently" sample the parameter space targeting the areas in the parameter space with highest error produced a more accurate ROM for the fixed number of snapshots in comparison to random snapshot generation.

\subsubsection{Surrogate Modeling:}

In order to construct a sparse grid approximation of the displacement with respect to the vector of unknown parameters and the vector of uncertain system parameters (parameters of the RBF, excitation frequency, and the location of the actuator) as described in Section 2.1, a 6 dimensional level 5 sparse grid, which required 1,345 evaluations of the ROM, was found to be suitable to approximate the displacement fields. Note that the computing cost (in terms of analysis time) of the ROM was approximately $10 \%$ of the cost of the equivalent full-order model, and therefore, the overall cost of creating the surrogate model was approximately $10 \%$ of what would have been required with a full-order model. 


\subsubsection{Material Characterization Results:}

The objective of the inverse problem for this first example was to determine the statistics of the elastic modulus distribution, and therefore the RBF parameters $(D, c$, and $\vec{\epsilon})$, with respect to the nondestructive test with the uncertain location of the actuation $\left(X_{F}\right)$. The objective functional used for the optimization-based inverse solution procedure in terms of the nine displacement measurement locations $\left(\left\{\vec{X}_{S i}\right\}_{i=1}^{9}\right)$ at the 10 excitation frequencies was cast as:

$$
J(D, c, \vec{\epsilon})=\sum_{i=1}^{9} \sum_{j=1}^{10} \frac{\left(R^{\text {mes }}\left(\vec{X}_{S i}, \omega_{j}\right)-R^{\operatorname{sim}}\left(\vec{X}_{S i}, \omega_{j} ; D, c, \vec{\epsilon}, X_{F}\right)\right)^{2}}{\left(R^{\text {mes }}\left(\vec{X}_{S i}, \omega_{j}\right)\right)^{2}},
$$

where $R^{\text {mes }}$ is the simulated experimentally measured response (i.e., target response) and $R^{\text {sim }}$ is the response estimate simulated with the surrogate model for a given estimate of the inverse problem solution and the uncertain parameter. To estimate the statistical moments of the inverse problem solution, the optimization-based solution procedure was repeated 20 times, each time with a different randomly generated value of the actuator location (within the given bounds) assumed within the surrogate model estimate of the system response to be compared to the simulated experimental measurements. Also note that the actuator location used to generate the simulated experimental measurements was randomly selected, but fixed for all trials (as would be the case in reality). 20 was chosen arbitrarily as the number of trials, since the accuracy of the statistics of the solution was considered less important than showing that the surrogate model approach was capable of estimating these solutions (nearly) as accurately as a traditional (full-order) model, but with a fraction of the computational expense. For each trial the stopping criteria for the GA optimization process to estimate the material parameters was set as a maximum of 7,000 functional evaluations.

Table 1 shows the mean (the first moment) and variance (the second central moment) of the RBF parameters estimated by the inverse solution process. Table 1 also shows the mean and variance of the measurement error corresponding to the parameter estimates with respect to the surrogate model (Equation 17) and the measurement error of those parameter estimates with respect to the full-order model (Equation 17 with the full-order model in place of the surrogate model for $R^{\text {sim }}$ ), as well as the error between the surrogate model and the full-order model (Equation 17 with the full-order model in 
Table 1: Target values for the unknown damage amplitude $(D)$, the breadth $(c)$, and the horizontal and vertical location of the damage center $\left(\epsilon_{x}, \epsilon_{y}\right)$, the mean and variance of the corresponding values estimated for the 20 trials, and the mean and variance of the measurement error corresponding to the parameter estimates with respect to the surrogate model (SM-EXP) and with respect to the full-order model (FOM-EXP), and the error between the surrogate model and the full-order model (SM-FOM) for the first example.

\begin{tabular}{l|cccc|ccc}
\multicolumn{2}{c}{$D$} & $c$ & $\epsilon_{x}$ & $\epsilon_{y}$ & \multicolumn{3}{c}{ Relative $L_{2}$-Error } \\
\hline Target Value & 0.7 & 0.005 & 0.7 & 0.3 & SM-EXP & FOM-EXP & SM-FOM \\
\hline Mean & 0.51 & 0.0049 & 0.7 & 0.36 & 0.12 & 0.18 & 0.018 \\
Variance & 0.062 & $9.18 \times 10^{-6}$ & 0.019 & 0.022 & 0.006 & 0.043 & 0.004 \\
\hline
\end{tabular}

place of the target response, $R^{\text {mes }}$ ) for the parameter estimates. The resulting parameter values were consistent with the sensitivity of the system response to the uncertain system parameter, with the mean value of the breadth and horizontal location of the RBF having been accurate in comparison with the target values (i.e., those used to simulate the experimental data) and with relatively low variances. Alternatively, the amplitude and vertical location of the RBF were more sensitive to the uncertain actuator location, particularly relative to the sensitivity of the measured response to those parameters themselves, and therefore, had significantly more relative variance and lower accuracy overall. One physical interpretation would be that the given test was considerably more reliable in predicting the horizontal location and size of the material variation in comparison to the magnitude and depth of the variation. The displacement measurement error with respect to the simulated experiment with the full-order model in place of surrogate model shows higher relative $L_{2}$-Error than the measurement error of simulated experiment with surrogate model, which is expected since the error objective functional was minimized based on surrogate model. More importantly, the difference between the surrogate and full-order models and the change in measurement error when analyzing the solutions with the full-order model rather than the surrogate model were both relatively low. These low errors provide confidence that the surrogate model was able to accurately represent the system response, and thus, provide accurate estimates to the statistical moments of the inverse problem solution for the elastic modulus distribution. This is particularly impressive when considering that the surrogate model was built using only 177 full-order analyses (127 for the ROM snapshots and 50 for the ROM test set) to then be used for 140,000 system evaluations $(7,000$ evalu- 


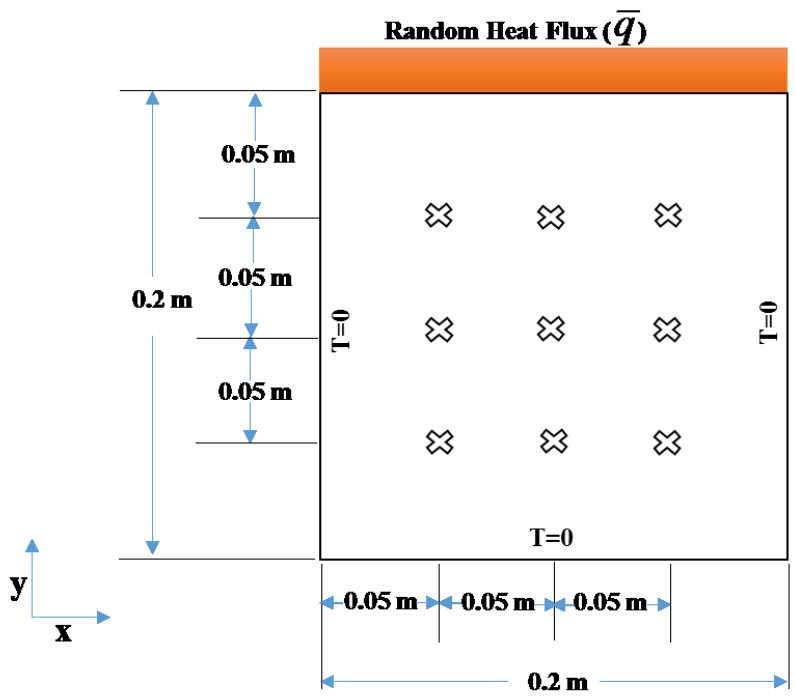

Figure 3: Schematic for the second example of conductivity characterization from thermal testing, displaying the sensor locations (x-marks) and the temperature and flux boundary conditions.

ations for each of the $20 \mathrm{GA}$ trials), with those 140,000 system evaluations only requiring on the order of 1 hour of computing time on a standard $\mathrm{PC}$ with a $2.00 \mathrm{GHz}$ processor and $4 \mathrm{~GB}$ RAM.

\subsection{Conductivity Characterization from Thermal Testing}

The second numerical example was based upon characterization of the thermal conductivity of a solid from temperature measurements. Figure 3 shows a schematic of the $0.2 m \times 0.2 m$ two-dimensional plate structure and the simulated thermal testing considered. The simulated thermal testing involved applying a heat flux to the top surface of the plate, with the plate having a known initial temperature of 0 throughout and the remaining three sides fixed at a temperature of 0 , and the transient temperature response at 9 discrete "sensors" within the plate was measured. The applied heat flux was assumed to be the main source of uncertainty for this problem, with this uniformly applied flux assumed to be defined and known for the purposes of the inverse problem as:

$$
\bar{q}=7 \times 10^{5} \times(1+\zeta) W / m^{2},
$$


where the uniformly distributed random variable $\zeta \sim U(0,1)$. Then, the test was simulated by randomly generating 40 realizations of the applied heat flux from the given distribution, and for each realization of the applied flux the resulting temperature was measured at 10 uniformly spaced points in time between $0 s$ and $1 s$ at each sensor to produce the first moment and second central moment of the temperature at each sensor and each time step with respect to the variable flux.

\subsubsection{Forward Problem and Reduced-Order Modeling:}

The structure for this example was assumed to have a nonlinear transient thermal response due to a nonlinear thermal conductivity and no internal heat sources, with the SPDE and boundary conditions governing the behavior given as:

$$
\begin{array}{r}
\rho C_{v} \frac{\partial T(\vec{x}, t, \vec{\varphi})}{\partial t}-\nabla \cdot[\kappa(T(\vec{x}, t, \vec{\varphi})) \cdot \nabla T(\vec{x}, t, \vec{\varphi})]=0, \quad \forall \vec{x} \in \Omega, \\
-(\kappa(T(\vec{x}, t, \vec{\varphi})) \cdot \nabla T(\vec{x}, t, \vec{\varphi})) \cdot \vec{n}(\vec{x})=\bar{q}(\zeta), \quad \forall \vec{x} \in \Gamma_{q}, \\
T(\vec{x}, t, \vec{\varphi})=0, \quad \forall \vec{x} \in \Gamma_{T}, \\
T(\vec{x}, t=0, \vec{\varphi})=0, \quad \forall \vec{x} \in \Omega,
\end{array}
$$

where $\mathrm{T}$ is the temperature, $t \in[0,1] s$ is the time, $\rho$ is the density, $C_{v}$ is the specific heat, $\kappa(T)$ is the temperature-dependent thermal conductivity, $\vec{\varphi}$ is again the vector of both the unknown/inverse problem parameters (heat conductivity parameters for this example) and the uncertain system parameters (heat flux magnitude for this example), $\vec{n}$ is the unit normal to the surface, $\Omega$ is the domain of the structure, $\Gamma_{q}$ is the portion of the domain boundary with applied heat flux boundary conditions, and $\Gamma_{T}$ is the portion of the domain boundary with applied temperature boundary conditions. The specific material parameters were based on those utilized in [5] (with some minor modification to the function used to define the thermal conductivity), with a density of $\rho=7850 \mathrm{~kg} / \mathrm{m}^{3}$ and specific heat of $C_{v}=419 \mathrm{~J} /\left(\mathrm{kg} \cdot{ }^{\circ} \mathrm{C}\right)$. The temperature-dependent thermal conductivity $\kappa(T)$ was assumed to be

the primary unknown of the inverse problem and defined in terms of four scalar coefficients in the following form:

$$
\kappa(T(\vec{x}, t, \vec{\varphi}))=c_{3} T^{3}(\vec{x}, t, \vec{\varphi})+c_{2} T^{2}(\vec{x}, t, \vec{\varphi})+c_{1} T(\vec{x}, t, \vec{\varphi})+c_{0} .
$$

For the ROM and surrogate model generation and the inverse characterization process, the four material parameters were assumed to be bounded as 
follows: $c_{0} \in[420,980] \mathrm{W} /(\mathrm{m} \mathrm{K}), c_{1} \in\left[1.8 \times 10^{-3}, 4.2 \times 10^{-3}\right] \mathrm{W} /(\mathrm{m} \mathrm{K})$, $c_{2} \in\left[1.2 \times 10^{-3}, 2.8 \times 10^{-3}\right] \mathrm{W} /(\mathrm{m} \mathrm{K})$, and $c_{3}=\left[0.66 \times 10^{-3}, 1.55 \times 10^{-3}\right]$ $\mathrm{W} /(\mathrm{m} \mathrm{K})$.

Applying the Galerkin projection described in Section 2.2 to the governing equations (Equation 19) produces the ROM for the nonlinear transient heat transfer problem as:

$$
\begin{array}{r}
\rho C_{v} \frac{\partial \alpha_{c}(\vec{\varphi}, t)}{\partial t}+c_{0} \sum_{i=1}^{m} \alpha_{i}(\vec{\varphi}, t) \int_{\Omega} \nabla \phi_{i}(\vec{x}) \cdot \nabla \phi_{c}(\vec{x}) d \vec{x} \\
+c_{1} \int_{\Omega}\left(\sum_{j=1}^{m} \alpha_{j}(\vec{\varphi}, t) \phi_{j}(\vec{x})\right) \cdot \sum_{i=1}^{m} \alpha_{i}(\vec{\varphi}, t) \nabla \phi_{i}(\vec{x}) \cdot \nabla \phi_{c}(\vec{x}) d \vec{x} \\
+c_{2} \int_{\Omega}\left(\sum_{j=1}^{m} \alpha_{j}(\vec{\varphi}, t) \phi_{j}(\vec{x})\right)^{2} \cdot \sum_{i=1}^{m} \alpha_{i}(\vec{\varphi}, t) \nabla \phi_{i}(\vec{x}) \cdot \nabla \phi_{c}(\vec{x}) d \vec{x} \\
+c_{3} \int_{\Omega}\left(\sum_{j=1}^{m} \alpha_{j}(\vec{\varphi}, t) \phi_{j}(\vec{x})\right)^{3} \cdot \sum_{i=1}^{m} \alpha_{i}(\vec{\varphi}, t) \nabla \phi_{i}(\vec{x}) \cdot \nabla \phi_{c}(\vec{x}) d \vec{x} \\
-\int_{\Gamma_{q}} \phi_{c}(\vec{x}) \bar{q} d \vec{x}=0, \quad \text { for } c=1 \ldots m,
\end{array}
$$

with

$$
\alpha_{c}(\vec{\varphi}, t=0)=\int_{\Omega} T_{0} \cdot \phi_{c}(\vec{x}) d \vec{x}, \quad \text { for } \quad c=1 \ldots m .
$$

The snapshot temperature fields generated with full-order analyses using the adaptive snapshot generation algorithm were based upon variations in the thermal conductivity parameters $\left(c_{0}, c_{1}, c_{2}\right.$, and $\left.c_{3}\right)$ and the heat flux amplitude parameter $(\zeta)$. Alternatively, for simplicity, the time sampling was fixed for the snapshot generation, with three times generated for each conductivity and heat flux combination: $t=0.3 \mathrm{~s}, t=0.6 \mathrm{~s}$, and $t=0.9 \mathrm{~s}$. Through initial testing of this example, these 3 snapshots through time were determined to be sufficient for approximating the system behavior. However, it should be noted that as the nonlinearity in time of a problem increases, the number of snapshots in time needed for accuracy will also increase. The adaptive nested grid snapshot generation algorithm converged at a total of 36 transient full-order analyses to form the 108 snapshots used to create the ROM. The final value of $\mathrm{L}$ at convergence was 1 , which produced 115 dimensional snapshot parameter sets for each value of time. An additional 25 
snapshot parameter sets were added during the refinement cycle ( 5 iterations with 5 parameter sets generated each iteration).

Again, an intermediate test was performed first, in which the response approximation capabilities of an ROM created with the adaptive strategy presented was compared to the approximation capabilities of a baseline ROM created with an equivalent number (i.e., 50) of snapshots that were entirely randomly generated from the space of input parameters. The average relative $L_{2}$-error and the maximum relative $L_{\infty}$-error between both $\mathrm{ROMs}$ and a set of 50 test response fields that were randomly generated based on quasirandom low-discrepancy Hammersley sampling method calculated. The two error values $\left(L_{2}\right.$ and $\left.L_{\infty}\right)$ for the adaptively generated ROM were $0.85 \%$ and $1.05 \%$, respectively, while the error values for the baseline ROM were $1.79 \%$ and $2.09 \%$, respectively. Thus, the use of the adaptive nested grid snapshot generation strategy improved the accuracy of the resulting ROM by $52 \%$ in terms of the average relative $L_{2}$-error and $50 \%$ in terms of the $L_{\infty}$-error in comparison to a common random generation approach for this second example.

\subsubsection{Surrogate Modeling:}

A 6 dimensional level 5 sparse grid was used to construct a suitably accurate surrogate model approximation of the temperature response with respect to the thermal conductivity parameters $\left(c_{0}, c_{1}, c_{2}\right.$, and $\left.c_{3}\right)$, the heat flux parameter $(\zeta)$, and the time $(\mathrm{t})$, which required 1,345 evaluations of the ROM. Similarly as the previous example, the computing cost of the ROM was approximately $5 \%$ compared to that of the full-order model used to create the ROM, and therefore, the overall cost of constructing the sparse grid surrogate model was $5 \%$ of the computing cost that would have been required to use the full-order model only.

\subsubsection{Material Characterization Results:}

The objective of the inverse problem for this second example was to determine the parameters of the temperature-dependent thermal conductivity function $\left(c_{0}, c_{1}, c_{2}\right.$ and $\left.c_{3}\right)$ with respect to the simulated thermal test that produced the two statistical moments (i.e., mean and variance) of the temperature at each sensor location $\left(\left\{\vec{X}_{S i}\right\}_{i=1}^{9}\right)$ at 10 uniformly spaced points in time between $0 s$ and $1 s\left(\left\{t_{i}\right\}_{i=1}^{10}\right)$ with respect to the uncertain applied flux parameter $(\zeta)$. Therefore, the objective functional used for the optimization- 
Table 2: Target (i.e., simulated experimental) values for the heat conductivity parameters $\left(c_{0}, c_{1}, c_{2}\right.$ and $\left.c_{3}\right)$ and the corresponding values estimated by the inverse characterization process, as well as the respective relative error for each estimated parameter.

\begin{tabular}{l|cccc}
\multicolumn{1}{c}{$c_{0}$} & $c_{1}$ & $c_{2}$ & $c_{3}$ \\
\hline Target Value & 650 & $2.5 \times 10^{-} 3$ & $2.5 \times 10^{-} 3$ & $1.2 \times 10^{-} 3$ \\
Estimated Value & 638 & $2.01 \times 10^{-} 3$ & $2.39 \times 10^{-} 3$ & $1.15 \times 10^{-} 3$ \\
\hline Relative Error & $1.8 \%$ & $19.6 \%$ & $4.4 \%$ & $4.2 \%$ \\
\hline \hline
\end{tabular}

based inverse solution procedure was defined as:

$$
J=\sum_{i=1}^{10} \sum_{j=1}^{10} \sum_{k=1}^{2}\left|\frac{\left\langle T^{m e s}\left(\vec{X}_{S i}, t_{j}\right)\right\rangle_{k}-\left\langle T^{s i m}\left(\vec{X}_{S i}, t_{j} ; c_{0}, c_{1}, c_{2}, c_{3}, \zeta\right)\right\rangle_{k}}{\left\langle T^{m e s}\left(\vec{X}_{S i}, t_{j}\right)\right\rangle_{k}}\right|
$$

where $T^{m e s}$ is the simulated experimentally measured response, $T^{\text {sim }}$ is the response estimate simulated with the surrogate model for a given estimate of the inverse problem solution and the uncertain parameter, $\langle\cdot\rangle_{1}$ represents the first moment operator, $\langle.\rangle_{2}$ represents the second central moment operator, and $|$.$| is the absolute value operator. Similarly to the approach that was used$ to generate the simulated experimental data, the moments of the temperature response were estimated with the surrogate model during the optimization process (i.e., at each iteration) by randomly generating 40 realizations of the applied heat flux parameter, evaluating the surrogate model with each realization (given values of the thermal conductivity parameters and the time), and then estimating the moments from the results. The stopping criteria for the GA optimization process to estimate the material parameters for this example was set as a maximum of 40,000 evaluations of the error functional.

Table 2 shows the thermal conductivity parameters used to simulate the experimental measurements (i.e., the target conductivity parameters), the thermal conductivity parameters estimated with the inverse solution process, and the relative error between each target and estimated parameter. More importantly, Figure 4 shows a plot of the temperature-dependent thermal conductivity that corresponds to the parameters used to simulate the experimental measurements (i.e., the target conductivity function) compared to the thermal conductivity corresponding to the parameters estimated through the inverse solution procedure. These results clearly indicate that the inverse 


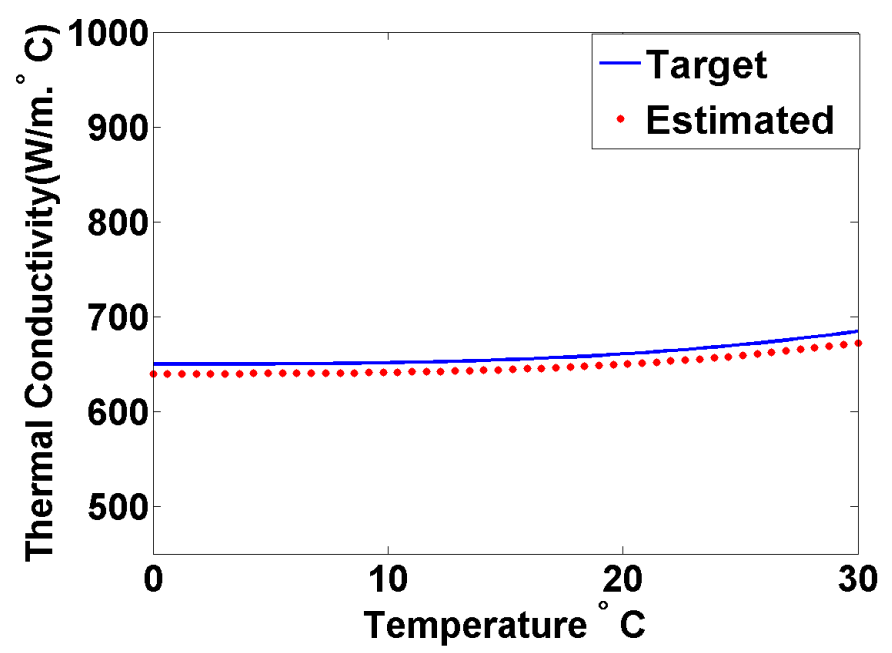

Figure 4: Target (i.e., simulated experimental) temperature-dependent thermal conductivity and the thermal conductivity estimated by the inverse characterization process (Estimated).

procedure utilizing the computationally efficient surrogate model was able to accurately estimate temperature-dependent thermal conductivity, with the approximate relative $L_{2}$-error in the thermal conductivity being less than $1 \%$. More specifically, the surrogate model was capable of successfully capturing the relationship between the temperature response and the uncertain applied flux for the potential variations in the thermal conductivity with sufficient accuracy to guide the optimization-based inverse solution procedure through the parameter space to an accurate solution. Similarly to the previous example, the surrogate model also provided a dramatic decrease in the computational expense compared to the cost of using the full-order model or reduced-order model directly. For this second example, the surrogate model was built using only 86 full-order analyses (36 for the ROM snapshots and 50 for the ROM test set) to then be used for 40,000 system evaluations, which required on the order of 30 minutes of computing time on a standard PC with a $2.00 \mathrm{GHz}$ processor and $4 \mathrm{~GB}$ RAM.

\section{Conclusion}

A novel approach was presented for creating a computationally efficient polynomial approximation (i.e., surrogate model) of a system response with 
respect to any designated unknown parameters, including parameters that may be considered to have significant uncertainty and/or parameters that are entirely unknown and sought to be determined through a nondestructive evaluation procedure. To enhance the overall efficiency of the approach, a novel algorithm was included as an intermediate step for creating a reducedbasis type reduced-order model of the system of interest. This intermediate step was based upon a technique to use nested grids to adaptively generate a data ensemble that is representative of the potential system response with respect to the unknown parameters. The overall approach would then use this computationally efficient ROM to create the surrogate model rather than a full-order model (e.g., traditional finite element analysis) at a substantial computational savings. This approach to generate an ROM was shown to provide a more accurate representation of the system of interest in comparison to a commonly used approach of randomly generating the response field ensemble. The overall surrogate modeling approach was then evaluated through numerically simulated example inverse problems based on characterization of material properties for two different systems, involving solid mechanics and heat transfer, respectively. Not only did the two examples consider different physical processes, but they also consider two different ways that uncertainty could be present and significant within NDE applications. The first example showed that the surrogate modeling approach could be used to computationally efficiently and accurately estimate the statistical moments of the parameters for an unknown stiffness distribution for a dynamically tested solid with uncertainty in the applied actuation. Lastly, the surrogate modeling approach was shown to be able to provide a single estimate, again both efficiently and accurately, of the parameters for an unknown temperature-dependent thermal conductivity for a solid in which the inverse problem objective was to match the statistical moments of the measured temperature field given an uncertain applied heat flux.

\section{Funding:}

The authors gratefully acknowledge the financial support of the National Science Foundation through Award No. 1130548. 
[1] Acharjee, S. and Zabaras, N. (2003). "A proper orthogonal decomposition approach to microstructure model reduction in rodrigues space with applications to optimal control of microstructure-sensitive properties." Acta Materialia, 51(18), 5627-5646.

[2] Aguilo, M., Aquino, W., Brigham, J., and Fatemi, M. (2010). "An inverse problem approach for elasticity imaging through vibroacoustics." Medical Imaging, IEEE Transactions on, 29(4), 1012-1021.

[3] Ahmadpoor, M., Notghi, B., and Brigham, J. C. (2016). "A generalized iterative approach to improve reduced-order model accuracy for inverse problem applications." Journal of Engineering Mechanics, 1943(7889), 04016020-1-04016020-12.

[4] Albanese, R., Banks, H., and Raye, J. (2002). "Nondestructive evaluation of materials using pulsed microwave interrogating signals and acoustic wave induced reflections." Inverse Problems, 18(6), 348-363.

[5] Aquino, W., Brigham, J., Earls, C., and Sukumar, N. (2009). "Generalized finite element method using proper orthogonal decomposition." International Journal for Numerical Methods in Engineering, 79(7), 887906.

[6] Arnst, M., Ghanem, R., Phipps, E., and Red-Horse, J. (2012). "Dimension reduction in stochastic modeling of coupled problems." International Journal for Numerical Methods in Engineering, 92(11), 940-968.

[7] Atwell, J. A. and King, B. B. (2001). "Proper orthogonal decomposition for reduced basis feedback controllers for parabolic equations." Mathematical and computer modelling, 33(1), 1-19.

[8] Audoin, B. (2002). "Non-destructive evaluation of composite materials with ultrasonic waves generated and detected by lasers." Ultrasonics, 40(1), 735-740.

[9] Babuška, I., Nobile, F., and Tempone, R. (2010). "A stochastic collocation method for elliptic partial differential equations with random input data." SIAM review, 52(2), 317-355. 
[10] Barthelmann, V., Novak, E., and Ritter, K. (2000). "High dimensional polynomial interpolation on sparse grids." Advances in Computational Mathematics, 12(4), 273-288.

[11] Berkooz, G., Holmes, P., and Lumley, J. L. (1993). "The proper orthogonal decomposition in the analysis of turbulent flows." Annual review of fluid mechanics, 25(1), 539-575.

[12] Boyaval, S., Le Bris, C., Lelièvre, T., Maday, Y., Nguyen, N. C., and Patera, A. T. (2010). "Reduced basis techniques for stochastic problems." Archives of Computational methods in Engineering, 17(4), 435-454.

[13] Brigham, J. and Aquino, W. (2009). "Inverse viscoelastic material characterization using pod reduced-order modeling in acoustic-structure interaction." Computer Methods in Applied Mechanics and Engineering, 198(9), 893-903.

[14] Brooks, S. (1958). "A discussion of random methods for seeking maxima." Operations Research, 6(2), 244-251.

[15] Bungartz, H.-J. and Griebel, M. (2004). "Sparse grids." Acta numerica, $13,147-269$.

[16] Burkardt, J. (2010). "The combining coefficient for anisotropic sparse grids." Interdisciplinary Center for Applied Mathematics 83 Information Technology Department, Virginia Tech, 1-14.

[17] Cawley, P. and Adams, R. D. (1979). "A vibration technique for nondestructive testing of fibre composite structures." Journal of Composite Materials, 13(2), 161-175.

[18] Chinesta, F., Ammar, A., Lemarchand, F., Beauchene, P., and Boust, F. (2008). "Alleviating mesh constraints: model reduction, parallel time integration and high resolution homogenization." Computer methods in applied mechanics and engineering, 197(5), 400-413.

[19] Deb, K., Pratap, A., Agarwal, S., and Meyarivan, T. (2002). "A fast and elitist multi objective genetic algorithm: Nsga-ii." Evolutionary Computation, IEEE Transactions on, 6(2), 182-197. 
[20] Donahue, M. M., Buzzard, G., Rundell, A. E., Jayaraman, A., and Hahn, J. (2009). "Parameter identification with adaptive sparse grid-based optimization for models of cellular processes." Methods in bioengineering: systems analysis of biological networks(Artech House, Boston/London, 2009).

[21] Ganapathysubramanian, B. and Zabaras, N. (2007). "Sparse grid collocation schemes for stochastic natural convection problems." Journal of Computational Physics, 225(1), 652-685.

[22] Ghanem, R. and Spanos, P. D. (2003). Stochastic finite elements: a spectral approach. DoverPublications. com.

[23] Goldberg, D. E. et al. (1989). Genetic algorithms in search optimization and machine learning, Vol. 412. Addison-wesley Reading Menlo Park.

[24] Han, S. and Feeny, B. (2003). "Application of proper orthogonal decomposition to structural vibration analysis." Mechanical Systems and Signal Processing, 17(5), 989-1001.

[25] Hao, Z., Notghi, B., Vandenbossche, J. M., and Brigham, J. C. (2015). "Optimal wave propagation-based nondestructive test design for quantitative damage characterization." Journal of Nondestructive Evaluation, $34(2), 1-15$

[26] Isukapalli, S., Roy, A., and Georgopoulos, P. (2000). "Efficient sensitivity/uncertainty analysis using the combined stochastic response surface method and automated differentiation: Application to environmental and biological systems." Risk Analysis, 20(5), 591-602.

[27] Khakhali, A., Nariman-Zadeh, N., Darvizeh, A., Masoumi, A., and Notghi, B. (2010). "Reliability-based robust multi-objective crashworthiness optimisation of s-shaped box beams with parametric uncertainties." International Journal of Crashworthiness, 15(4), 443-456.

[28] Khalil, M., Adhikari, S., and Sarkar, A. (2007). "Linear system identification using proper orthogonal decomposition." Mechanical Systems and Signal Processing, 21(8), 3123-3145.

[29] Leibfritz, F. and Volkwein, S. (2006). "Reduced order output feedback control design for pde systems using proper orthogonal decomposition and 
nonlinear semidefinite programming." Linear algebra and its applications, $415(2), 542-575$.

[30] Lucia, D. J., Beran, P. S., and Silva, W. A. (2004). "Reduced-order modeling: new approaches for computational physics." Progress in Aerospace Sciences, 40(1), 51-117.

[31] Luenberger, D. (1997). Optimization by vector space methods. WileyInterscience.

[32] Ma, X. and Zabaras, N. (2009). "An efficient bayesian inference approach to inverse problems based on an adaptive sparse grid collocation method." Inverse Problems, 25(3), 035013.

[33] Marzouk, Y. and Xiu, D. (2009). "A stochastic collocation approach to bayesian inference in inverse problems." Communication in Computational Physics, 6(4), 826-847.

[34] Marzouk, Y. M. and Najm, H. N. (2009). "Dimensionality reduction and polynomial chaos acceleration of bayesian inference in inverse problems." Journal of Computational Physics, 228(6), 1862-1902.

[35] Narayanan, V. A. B. and Zabaras, N. (2004). "Stochastic inverse heat conduction using a spectral approach." International Journal for Numerical Methods in Engineering, 60(9), 1569-1593.

[36] Niederreiter, H. (2010). Quasi-Monte Carlo Methods. Wiley Online Library.

[37] Nocedal, J. and Wright, S. (1999). Numerical optimization. Springer verlag.

[38] Notghi, B. and Brigham, J. C. (2013). "Optimal nondestructive test design for maximum sensitivity and minimal redundancy for applications in material characterization." Smart Materials and Structures, 22(12), 125036 .

[39] Notghi, B. and Brigham, J. C. (2015). "A computational approach for robust nondestructive test design maximizing characterization capabilities for solids and structures subject to uncertainty." International Journal for Numerical Methods in Engineering, 104(4), 297-311. 
[40] Ogi, H., Sato, K., Asada, T., and Hirao, M. (2002). "Complete mode identification for resonance ultrasound spectroscopy." The Journal of the Acoustical Society of America, 112, 2553.

[41] Ostrowski, Z., Białecki, R. A., and Kassab, A. J. (2005). "Estimation of constant thermal conductivity by use of proper orthogonal decomposition." Computational Mechanics, 37(1), 52-59.

[42] Reddy, J. N. (1993). An introduction to the finite element method, Vol. 2. McGraw-Hill New York.

[43] Rozza, G., Huynh, D., and Patera, A. T. (2008). "Reduced basis approximation and a posteriori error estimation for affinely parametrized elliptic coercive partial differential equations." Archives of Computational Methods in Engineering, 15(3), 229-275.

[44] Sarkar, A., Benabbou, N., and Ghanem, R. (2009). "Domain decomposition of stochastic pdes: theoretical formulations." International Journal for Numerical Methods in Engineering, 77(5), 689-701.

[45] Sivanandam, S. and Deepa, S. (2007). Introduction to genetic algorithms. Springer Publishing Company, Incorporated.

[46] Smolyak, S. A. (1963). "Quadrature and interpolation formulas for tensor products of certain classes of functions." Dokl. Akad. Nauk SSSR, Vol. 4, 111.

[47] Soize, C. and Ghanem, R. (2004). "Physical systems with random uncertainties: chaos representations with arbitrary probability measure." SIAM Journal on Scientific Computing, 26(2), 395-410.

[48] Tatang, M. A. (1995). "Direct incorporation of uncertainty in chemical and environmental engineering systems." Ph.D. thesis, Massachusetts Institute of Technology, Massachusetts Institute of Technology.

[49] Trefethen, L. N. (2008). "Is gauss quadrature better than clenshawcurtis?." SIAM review, 50(1), 67-87.

[50] Wang, M. and Brigham, J. C. (2014a). "Assessment of multi-objective optimization for nondestructive evaluation of damage in structural components." Journal of Intelligent Material Systems and Structures, 25(9), 1082-1096. 
[51] Wang, M. and Brigham, J. C. (2014b). "A computational nondestructive evaluation algorithm combining self-evolving parameterization and multiobjective optimization for quantitative damage characterization." Journal of Nondestructive Evaluation, 33(4), 547-561.

[52] Wasilkowski, G. W. and Wozniakowski, H. (1995). "Explicit cost bounds of algorithms for multivariate tensor product problems." Journal of Complexity, 11(1), 1-56.

[53] Wong, T.-T., Luk, W.-S., and Heng, P.-A. (1997). "Sampling with hammersley and halton points." Journal of graphics tools, 2(2), 9-24.

[54] Xiu, D. and Hesthaven, J. (2005). "High-order collocation methods for differential equations with random inputs." SIAM Journal on Scientific Computing, 27(3), 1118-1139.

[55] Zabaras, N. and Ganapathysubramanian, B. (2008). "A scalable framework for the solution of stochastic inverse problems using a sparse grid collocation approach." Journal of Computational Physics, 227(9), 46974735 . 